\title{
Motility and $\beta$-Lactamases: Occurrences of Antibiotic Resistance in Nosocomial Infections
}

\section{Tejpreet Chadha*}

Department of Biological Sciences, Texas Tech University, Lubbock, Texas, USA

\begin{abstract}
The widespread use of $\beta$-lactam antibiotics has resulted in the global emergence of antibiotic-resistant bacterial strains. Multidrug resistant isolates are now more difficult to treat due to multiple intrinsic and acquired mechanisms of drug resistance that operate simultaneously in a bacterial cell. Motility is associated with virulence that contributes to multidrug resistance. The current analysis has shown that natural pattern of distribution of Ambler (molecular) classes of $\beta$-lactamases (A, B, C, D) varied among motile and non-motile nosocomial pathogens. Our results revealed that the relative abundance of class $A \beta$-lactamases was highest in bacterial species that are non-motile. However, the relative abundance of class $C \beta$-lactamases was mostly same for both motile and non-motile bacterial species. The class $D$-lactamases was found highest in bacterial species that are non-motile. However, based upon the total number of sequences checked, the prevalence of class B $\beta$-lactamases dominated in pathogens that are motile when compared to other Ambler classes of $\beta$-lactamases. Our results imply that occurrence of class $B$-lactamases may prove advantageous to motile bacterial species. This may also suggest that the gain of class $B$-lactamases genes during the course of their evolution may have contributed to their virulence.
\end{abstract}

Keywords: $\beta$-lactamases; Motility; Antibiotic resistance

\section{Introduction}

Antibiotics are essential for the treatment of bacterial infections in humans and animals [1]. The excessive use and misuse of antibiotics has resulted in economic losses to the consumer, hospital, and society, due to use of more expensive drugs for a second-line treatment, more tests and much longer stays in the hospital [2]. The excessive use of antimicrobial agents in a hospital setting has resulted in continued evolution of pathogens with multi-drug resistance. The several commensal organisms that commonly existed on the skin and the alimentary tract have now emerged as nosocomial pathogens [3-5]. This has seriously compromised ability to treat nosocomial infections. The common six pathogen that cause the majority of hospital-acquired infections in the United States, and are resistant to antibacterial drugs [6-9] are Enterococcus faecium, Staphylococcus aureus, Klebsiella pneumonia, Acinetobacter baumanii, Pseudomonas aeruginosa, and Enterobacter species [7]

Multi-drug resistance is considered to reflect multiple mechanisms operating simultaneously in a bacterial cell [10]. The multidrug-resistant bacteria have been discovered to contain newer $\beta$-lactamases, extendedspectrum $\beta$-lactamases (ESBLs), which are capable of hydrolyzing penicillin, broad-spectrum cephalosporin and monobactams [11] . With fewer drugs effective against such pathogens, the mortality rate is exceptionally high among persons with prolonged hospitalization and those who are critically ill and exposed to invasive devices such as ventilators or central venous catheters $[12,13]$. Motility has been counted as an important virulence factor and is often closely linked by complex regulatory networks [14]. The consequent loss of motility is mostly associated with decrease in virulence. The study by Gooderham et al. [15] have shown that single polar flagellum of Pseudomonas aeruginosa is an important and highly regulated virulence factor involved in both motility and surface attachment [15]. P. aeruginosa is known to cause serious antibiotic resistant infections such as cystic fibrosis [15]. The study by Kim et al. [16] have shown that swarming motility in Salmonella may bring in a unique physiological state that could equip cells to withstand high levels of antimicrobial compounds generated by the hosts [16]. The pili and flagella are important for the early stages of biofilm formation that further contribute to multi-drug resistance. The study by Gallant et al. [17] have shown that class A and $\mathrm{D} \beta$-lactamases inhibit biofilm formation. However, the class $\mathrm{B}$ or $\mathrm{C}$ $\beta$-lactamases do not decrease biofilm formation. There was a proposed model that $\beta$-lactamases may interfere with correct assembly of large macromolecular complexes participating in surface attachment and subsequent biofilm development [17].

Currently, we do not have any information on distribution of $\beta$-lactamases in motile and non-motile bacterial species. The evaluation of natural pattern of occurrences of $\beta$-lactamases will be a significant step toward understanding the distribution of $\beta$-lactamases in these isolates. Future study could further exploit the association of bacterial motility to a specific class of $\beta$-lactamases. This could further help to engineer a specific therapeutic antibacterial target to cure or prevent diseases.

\section{Material and Methods}

\section{Databases}

All information evaluated for this study was obtained from the National Center for Biotechnology Information (NCBI) Database [http://www.ncbi.nlm.nih.gov/] [18,19], and the Department of Energy (DOE), Joint Genome Institute (JGI) database [http://www.jgi.doe. gov/]. The bacterial species were further evaluated for motility and presence of the four different Ambler classes of $\beta$-lactamase genes (A, $B, C$ and $D$ ).

${ }^{*}$ Corresponding author: Tejpreet Chadha, Department of Biological Sciences, Texas Technological University, Lubbock, Texas USA, E-mail: preeti.chadha@ttu.edu

Received January 11, 2013; Accepted February 14, 2013; Published February 20, 2013

Citation: Chadha T (2013) Motility and $\beta$-Lactamases: Occurrences of Antibiotic Resistance in Nosocomial Infections. J Data Mining Genomics Proteomics 4: 126. doi:10.4172/2153-0602.1000126

Copyright: ( $\odot 2013$ Chadha T. This is an open-access article distributed under the terms of the Creative Commons Attribution License, which permits unrestricted use, distribution, and reproduction in any medium, provided the original author and source are credited. 
Data acquisition and organization: The genome browser using JGI database [http://img.jgi.doe.gov/cgi-bin/w/main.cgi] provides a list of all sequenced genomes under the categories domain: $\mathrm{B}=$ Bacteria, $\mathrm{A}=$ Archaea, $\mathrm{E}=$ Eukarya, $\mathrm{P}=$ Plasmids, $\mathrm{G}=$ Genome, Fragment, $\mathrm{V}=$ Viruses; and Genome Completion: F=Finished, $\mathrm{P}=$ Permanent Draft, $\mathrm{D}=$ Draft. The genomes that were under Domain Bacteria were selected for analysis. Once the list was compiled, bacterial information was organized on the basis of Gram stain reaction. The list was further organized by phylum, class, NCBI ID and occurrence of four different molecular classes of $\beta$-lactamases. The NCBI and JGI databases were used to compile the list of bacterial species whose complete genomes have been sequenced to date. The bacterial species that have $\beta$-lactamases were further evaluated for presence of the four different Ambler classes of $\beta$-lactamase genes (A, B, C and D).

\section{Data analysis}

The absolute numbers of species with positive $\beta$-lactamase status of Gram-negative bacteria were determined. To correct for differences in the total number of bacteria sequenced within a particular group relative abundances of the $\beta$-lactamases were calculated. The absolute abundance was calculated as per the total number of bacteria that were shown to have $\beta$-lactamase sequences within a taxonomic group. The proportional abundance was calculated by the total number of bacteria that were shown to have $\beta$-lactamase sequences divided by the total number of bacteria within a taxonomic group. The relative abundance was calculated by the total number of bacteria that were shown to have $\beta$-lactamase sequences divided by the total number of bacteria in for each Ambler class within a taxonomic group.

\section{Results}

The analysis included bacterial species that are known to cause nosocomial infection (Appendix-A). The list available for analysis was compiled with a definitive NCBI Taxon ID. The distribution of the four different Ambler (molecular) classes of $\beta$-lactamases (A, B, C, and D) varied among different bacterial species. The absolute abundance data showed that the prevalence of all the classes of $\beta$-lactamases was highest in non-motile as compared to motile bacterial species. The relative abundance of class A $\beta$-lactamases was highest in bacterial species that are non-motile (Figure 1) in comparison to non-motile bacterial species. However, class C $\beta$-lactamases was same for both motile and non-motile bacterial species (Figure 1). The relative abundance of class D $\beta$-lactamases was highest in bacterial species that are non-motile (Figure 1) in comparison to motile bacterial species. Based upon the total number of sequences checked, the prevalence of class B $\beta$-lactamases dominated in pathogens that are motile when compared to other Ambler classes of $\beta$-lactamases (Figure 3) within motile bacterial species. However, absolute abundance of class $\mathrm{C}$ $\beta$-lactamases was highest among non-motile bacterial species (Figure 3 ). The absolute abundance of class $\mathrm{D} \beta$-lactamases was prevalent among non-motile pathogens (Figure 3 ). The proportional abundance data provided evidence of occurrence of all four molecular classes of $\beta$-lactamases across motile and non-motile pathogens (Figure 2). The relative abundance of class $B \quad \beta$-lactamases was highest in both motile and non-motile pathogens when compared to the other molecular classes of $\beta$-lactamases individually (Figure 1).

\section{Discussion}

The current distribution of $\beta$-lactamases may reflect on-going adaptation of ecological traits that could arise by recent adaptive responses of the organisms to the current environments. In addition

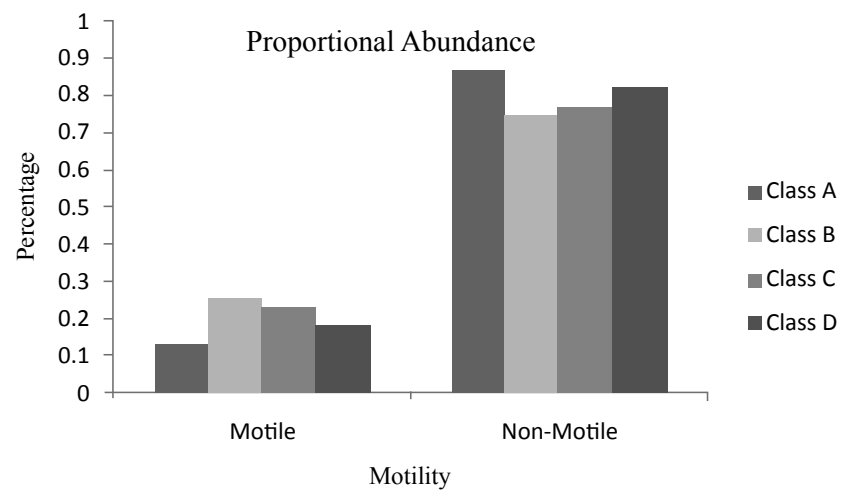

Figure 1: Relative abundances of the total $\beta$-lactamase occurrences by molecular class and by motility for all bacterial species. Molecular classes are defined as in figure 3 .

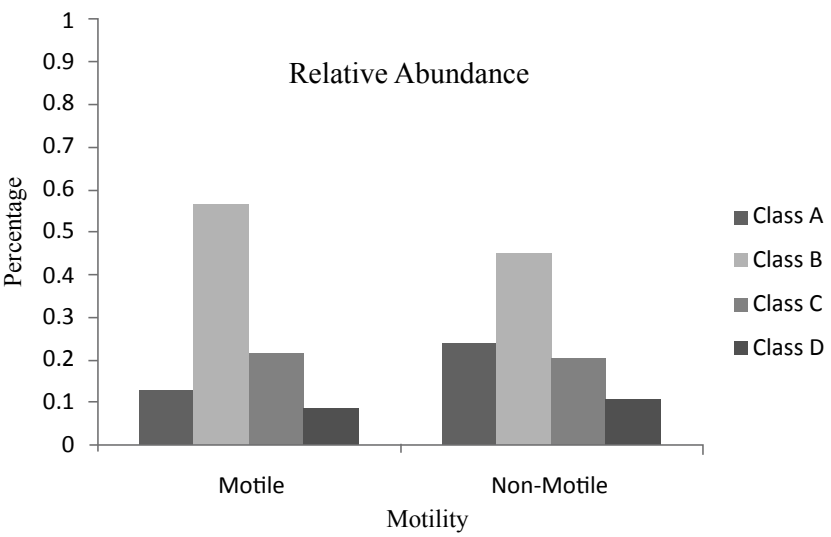

Figure 2: Proportional abundances of the total $\beta$-lactamase occurrences by molecular class and by motility for all bacterial species. Molecular classes are defined as in figure 3 .

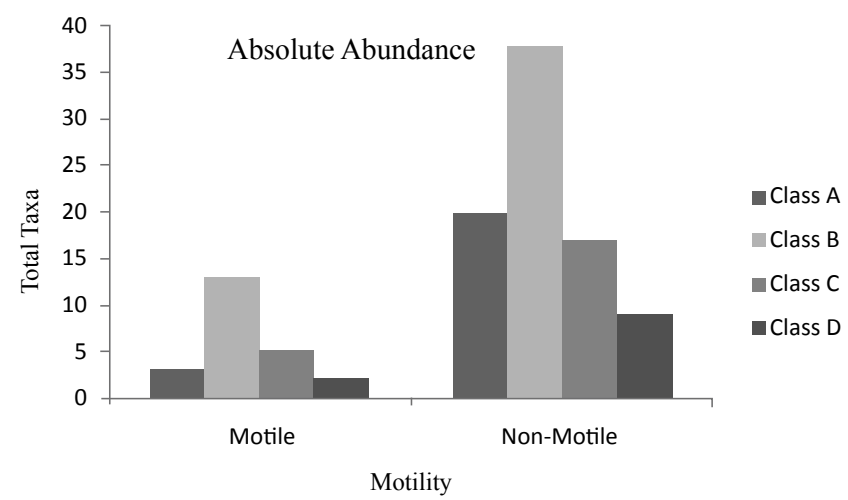

Figure 3: Distribution of the total $\beta$-lactamase occurrences by molecula class and by motility for bacterial species (Appendix-A). Class stands for the Ambler classification of $\beta$-lactamases, which divides these enzymes into four molecular classes, $A, B, C$, and $D$, based on their conserved and unique amino acid motifs. Class $A, C$, and $D$ are serine $\beta$-lactamases and Class $B$ is metallo$\beta$-lactamases. 
to understanding the basic patterns of $\beta$-lactamase distributions according to motility, the data presented in this investigation have real importance as it provides an assessment of the possible roles of resistance genes in natural microbial communities and their indirect and direct linkages to their occurrences in clinical settings. The past evolution of serine $\beta$-lactamases has shown that the Class A $\beta$-lactamases has been around for a very long time. They have been moving onto plasmids, from plasmids into chromosomes, moving among species and even across bacterial phyla $[20,21]$. The Class D $\beta$-lactamases are a diverse group of enzymes whose genes are located both on plasmids and on the chromosomes of a wide range of Gram-negative bacteria [22]. Metallo- $\beta$-lactamases (MBLs), Class B $\beta$-lactamases, have gained clinical significance because of their mobility. They can be found as gene cassettes carried by integrons or transposons. The presence of Class B $\beta$-lactamase genes on integrons has resulted in their spread to other nosocomial pathogens in the hospital setting such as Klebsiella species [23]. The presence of $\beta$-lactamases can be identified as an important trait as they may play vital physiological roles such as selfprotection that may dictate whether a bacterial species can occur in a specific habitat [24]. In the current investigation high occurrence of class B $\beta$-lactamases suggests that these genes support functions other than antibiotic-resistance mechanisms which appear to respond to environmental heterogeneity. The studies have shown that class $\mathrm{A}$ and $\mathrm{D} \beta$-lactamases from plasmid vectors can negatively impact biofilm formation by Gram-negative bacteria [17] and at the same time conjugative resistance plasmids can enhance biofilm formation [25]. The studies have shown that bacteria may gain resistance to some antibiotics at the expense of other protective phenotypes such as the ability to form biofilms. The occurrence of $\beta$-lactamases may prove advantageous to bacterial species. However, this may also imply that horizontal acquisition of class B $\beta$-lactamase could have an effect on bacteria phenotype. Though, once these new genes are amplified in a population and selection pressure continues, there is likely a high probability that they will be moved into clinical settings simply by random events. This further suggests that the gain of $\beta$-lactamases genes during the course of their evolution may have contributed to their virulence.

\section{Acknowledgement}

The sequence data were obtained from National Center for Biotechnology Information database website (http://www.ncbi.nlm.nih.gov/) and the Joint Genome Institute (JGI) database [http://www.jgi.doe.gov/]. I would also like to thank $\mathrm{Dr}$ Randall Jeter, Dr. Alex Trindade, and Dr. John.C. Zak.

\section{References}

1. Allen HK, Donato J, Wang HH, Cloud-Hansen KA, Davies J, et al. (2010) Call of the wild: antibiotic resistance genes in natural environments. Nat Rev Microbiol 8: 251-259.

2. ReAct (2008) Economic aspects of antibiotic resistance. In ReAct, A fact sheet from ReAct - Action on Antibiotic Resistance, (1stedn) Uppsala University, Uppsala, Sweden.

3. Alekshun MN, Levy SB (2006) Commensals upon us. Biochem Pharmacol 71 893-900.

4. Goossens H, Grabein B (2005) Prevalence and antimicrobial susceptibility data for extended-spectrum beta-lactamase- and AmpC-producing Enterobacteriaceae from the MYSTIC Program in Europe and the United States (1997-2004). Diagn Microbiol Infect Dis 53: 257-264.
5. Riedel S, Beekmann SE, Heilmann KP, Richter SS, Garcia-de-Lomas J, et al. (2007) Antimicrobial use in Europe and antimicrobial resistance in Streptococcus pneumoniae. Eur J Clin Microbiol Infect Dis 26: 485-490.

6. Rice LB (2008) The Maxwell Finland Lecture: for the duration-rational antibiotic administration in an era of antimicrobial resistance and clostridium difficile. Clin Infect Dis 46: 491-496.

7. Rice LB (2008) Federal funding for the study of antimicrobial resistance in nosocomial pathogens: no ESKAPE. J Infect Dis 197: 1079-1081.

8. Rice LB (2000) Therapeutic considerations in the treatment of respiratory infections caused by ceftazidime-resistant Klebsiella pneumoniae. Semin Respir Crit Care Med 21: 323-329.

9. Rice LB, Carias LL, Hujer AM, Bonafede M, Hutton R, et al. (2000) High-leve expression of chromosomally encoded SHV-1 beta-lactamase and an outer membrane protein change confer resistance to ceftazidime and piperacillintazobactam in a clinical isolate of Klebsiella pneumoniae. Antimicrob Agents Chemother 44: 362-367.

10. Wright GD, Sutherland AD (2007) New strategies for combating multidrugresistant bacteria. Trends Mol Med 13: 260-267.

11. Gniadkowski M (2008) Evolution of extended-spectrum beta-lactamases by mutation. Clin Microbiol Infect 14: 11-32.

12. Hidron Al, Edwards JR, Patel J, Horan TC, Sievert DM, et al. (2008) NHSN annual update: antimicrobial-resistant pathogens associated with healthcareassociated infections: annual summary of data reported to the National Healthcare Safety Network at the Centers for Disease Control and Prevention, 2006-2007. Infect Control Hosp Epidemiol 29: 996-1011.

13. Lode $H$ (2005) Management of serious nosocomial bacterial infections: do current therapeutic options meet the need?. Clin Microbiol Infect 11: 778-787.

14. Josenhans C, Suerbaum S (2002) The role of motility as a virulence factor in bacteria. Int J Med Microbiol 291: 605-614

15. Gooderham WJ, Hancock RE (2009) Regulation of virulence and antibiotic resistance by two-component regulatory systems in Pseudomonas aeruginosa. FEMS Microbiol Rev 33: 279-294.

16. Kim W, Surette MG (2003) Swarming populations of Salmonella represen a unique physiological state coupled to multiple mechanisms of antibiotic resistance. Biol Proced Online 5:189-196.

17. Gallant CV, Daniels C, Leung JM, Ghosh AS, Young KD, et al. (2005) Common beta-lactamases inhibit bacterial biofilm formation. Mol Microbiol 58: 1012 1024

18. Federhen S (2012) The NCBI Taxonomy database. Nucleic Acids Res 40 D136-143.

19. Benson DA, Karsch-Mizrachi I, Lipman DJ, Ostell J, Sayers EW (2013) GenBank. Nucleic Acids Res 39: D32-37.

20. Pitout JD, Nordmann P, Laupland KB, Poirel L (2005) Emergence of Enterobacteriaceae producing extended-spectrum beta-lactamases (ESBLs) in the community. J Antimicrob Chemother 56: 52-59.

21. Hall BG, Barlow M (2004) Evolution of the serine beta-lactamases: past present and future. Drug Resist Updat 7: 111-123.

22. Hall BG, Barlow M (2003) Structure-based phylogenies of the serine betalactamases. J Mol Evol 57: 255-260.

23. Walsh TR, Toleman MA, Poirel L, Nordmann P (2005) Metallo-beta-lactamases: the quiet before the storm?. Clin Microbiol Rev 18: 306-325

24. Martinez JL (2009) The role of natural environments in the evolution of resistance traits in pathogenic bacteria. Proc Biol Sci 276: 2521-2530.

25. Ghigo JM (2001) Natural conjugative plasmids induce bacterial biofilm development. Nature 412: 442-445 